\title{
TREATMENT OF NEUROSYPHILIS BY ARSPHENAMIN INTRAVENOUSLY AND AUTO-ARSPHENAMINIZED SERUM INTRASPINALLY*
}

\author{
GEORGE M. GOODWIN, M.D., AND J. R. SCOTT, M.D. \\ NEW YORK
}

For the past seven years cases of syphilis of the central nervous system admitted to Dr. S. W. Lambert's service at St. Luke's Hospital have been treated with arsphenamin intravenously and the intraspinal administration of auto-arsphenaminized serum. About fifty such cases have received this treatment during this period, the majority of which have been of the tabetic type. The twenty-one cases reported herein have been investigated and followed up to determine the efficacy of this type of treatment.

\section{TECHNIC OF ADMINISTRATION}

The technic employed is essentially that of Swift and Ellis as described in their original article on the subject. Neoarsphenamin has been the drug usually administered intravenously; the other products, such as neodiarsenol, arsenobenzol, and novarsenobenzol, have been used when the former drug became difficult to obtain. Nine decigrams of neoarsphenamin were administered intravenously. One hour later sufficient blood was withdrawn to obtain from 10 to 30 c.c. of serum. The serum thus obtained was centrifuged for one-half hour and inactivated at $56 \mathrm{C}$. for one hour and injected intraspinally the following day. In the early cases this serum was diluted with saline solution to 40 per cent., but since then the concentrated serum has been used.

\section{REACTIONS OFTEN FOLLOW}

Reaction to the intraspinal treatment has varied a great deal. The usual reaction has consisted of darting pains in the legs, beginning an hour or two after the introduction of the serum, and lasting from twelve to seventy-two hours. Associated with these pains there has sometimes been severe headache, girdle sensations, and disturbances of bladder control. The reaction in one patient (Case 3 in the table) was unusually severe. This patient, several hours after the intraspinal treatment, suffered from intense headache and severe lightning pains and loss of bladder control. His temperature rose to $101.2 \mathrm{~F}$.,

* From Medical Division B, St. Luke's Hospital, New York. 
and he developed rigidity and retraction of the neck and a positive Kernig's sign. The following morning his white blood count rose to 31,000 , with 95 per cent. of polymorphonuclear cells in the smear, and the cell count in the spinal fluid was 10,000 , with 85 per cent. of polymorphonuclears. Cultures and smears of the spinal fluid were negative bacteriologically. Symptoms continued severe for five days and gradually abated. In a week the spinal fluid cell count fell to 75, and a month later it was 10 , with 100 per cent. lymphocytes in the smear, and the patient was free from all pain or other after effects.

While in some instances very little discomfort is experienced, it is usual to have more or less discomfort for twelve or more hours following the intraspinal treatment. For this reason the following medication is ordered as a routine measure after such treatments:

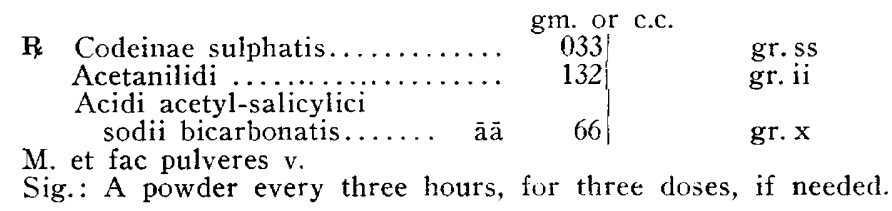

We have been unable to demonstrate any relationship between the severity of the reactions and the dilution, or quantity of the serum injected, in amounts between 10 and 25 c.c. We are convinced, however, that cloudy serums due to the presence of an excess of fat or to hemoglobin are much more likely to give severe reactions than are clear serums. The presence of hemoglobin can be guarded against in part by the use of dry syringes, needles, and flasks, and care in seeing that the blood is not shaken up in transit to the laboratory. The presence of an excess of fat is more difficult to control, but the serums may be improved in this respect by having the patients fast for five or more hours before the blood is to be withdrawn.

\section{SYMPTOMS IN THIS SERIES OF CASES}

In the accompanying table the data concerning the symptoms presented in these cases have been recorded under three main heads: subjective and objective symptoms, and laboratory evidence. Subjectively, the most common symptom has been pain ; after this, disturbances in gait; then disturbances in bladder control and vertigo. Objectively, 85 per cent. have shown pupillary disturbances, 76 per cent. a Romberg sign, and 52 per cent. an absence of knee jerks. Seventy-six per cent. have given positive blood Wassermann reactions, 76 per cent. positive spinal fluid Wassermann reactions, and 76 per cent. have shown an increase in globulin. Three cases gave positive spinal fluid Wassermann reactions and negative blood Wassermann reactions, and two cases showed the contrary condition. 


\section{RESULTS OF TREATMENT}

It cannot be expected of any form of therapy that it will undo destructive changes which have already occurred. But this form of treatment may be expected to control associated meningeal inflammations. The control of an associated meningeal involvement is the best explanation we can give for the improvement which these cases have shown.

We have observed no instances among well defined tabetic cases where absent knee jerks have returned, or where sluggish pupils have regained their activity. We have been impressed by the frequent improvement in the ataxia of these cases as manifested by increased steadiness in gait and a decrease in the intensity of the Romberg sign. These cases almost uniformly show improvement in nutrition, several of them showing increases in weight varying from 16 to 31 pounds.

The most gratifying results from the treatment have been shown by its effect on the subjective symptoms. In spite of the fact that the intraspinal treatments often cause severe reactions we have found these patients more anxious to continue their treatments and to return to the hospital than any other class.

From this point of view, also, it is not usual to see a return to the condition of normality, but we have been struck in questioning these patients with the fact that in most of the cases there has been a very marked and sustained diminution in the amount of discomfort from which they were suffering before the institution of treatment. In almost every one of the cases here recorded there has been relief to a great extent from pain. Similarly there has been a frequent improvement in bladder function. Of six patients suffering from vertigo, in four the condition has disappeared under treatment, one has shown improvement, and in the sixth, the condition has become progressively worse in spite of vigorous treatment.

The influence of the treatment on the serology and cytology of the disease can best be seen by reference to the table. Here it will be seen that the blood Wassermann reaction has either been reduced in degree or changed to a negative reaction in all but two cases, in which it was uninfluenced. The spinal fluid Wassermann reaction has been reduced in degree or changed to a negative reaction in nine instances, has been uninfluenced in five cases, and in one case has become stronger during treatment. The globulin reaction has been reduced in thirteen cases, and has become stronger in five. Since the introduction of the colloidal gold reaction as a routine test in cases of nervous disease eight of the cases of this series have been tested by this means on each of their admissions. Two cases have shown positive gold curves in the absence of either a positive blood or spinal fluid Wassermann 
reaction. One case has shown a positive curve with a negative spinal fluid Wassermann reaction, but a positive blood Wassermann reaction. All of the cases with positive curves have shown increases in the spinal fluid cell count and globulin content. Seven of the cases have shown a decrease in the intensity of the reaction after treatment. In the eighth case the curve assumed the paretic type during treatment and the patient developed general paresis clinically (Case 20).

As we have noted above, the majority of the cases were of the tabetic type. Two of the cases were spastic in type. One of these showed marked improvement after fifteen treatments with the disappearance of spasticity on the affected side, and a return of the reflexes to normal (Case 15). In another similar case of longer duration the patient has shown moderate improvement after fourteen treaments, and can walk with the use of a cane (Case 17). We have not undertaken the treatment of any paretic cases, but have had two cases develop paresis during or after treatment. One of these patients had received six treatments over a period of four months, the other had been under treatment for tabes for twenty-four months and had received seventeen treatments (Cases 20 and 21 ).

We have endeavored in the accompanying table to state graphically the essential data concerning the cases, using the plus sign to indicate the presence of a condition, and the zero sign to denote its absence, and using one, two, three, or four plus signs according to the relative intensity of the symptom.

We have selected the following group of cases to discuss in greater detail:

\section{ILLUSTRATIVE CASES}

CASE 3.-History.-F. S., a man, aged 48, had symptoms during a period of three years. He has had attacks of severe shooting pains in legs at irregular intervals, with difficulty in walking in the dark.

Physical Examination.-This revealed unimpaired sensations, equal but sluggish pupils, absent knee jerks, slight ataxia of legs, and positive Romberg sign.

Laboratory Findings.-The blood Wassermann reaction was positive; spinal fluid Wassermann reaction, positive; cell count, 4.

Treatment.-This patient had received one or two intravenous injections of arsphenamin before admission to the hospital. After admission he received two injections intravenously of $0.9 \mathrm{gm}$. of neoarsphenamin and two intraspinal injections of 40 per cent. serum. After the second injection he developed the severe reaction described in the beginning of this article. He was discharged from the hospital in March, 1913, and went to Alabama to live.

Results.-Three years later in reply to our inquiry his physician, Dr. F. G. DuBose of Selma, Ala., writes: "The patient has had six intravenous injections of arsphenamin since his discharge and injections of bichlorid of mercury in the intervals. These were given in the first year after leaving the hospital. Four blood Wassermann reactions in the past year have been negative. For nine months after leaving the hospital he suffered at intervals with pains in the calves of the legs. At the present time he is free from pain or other tabetic symptoms. No Romberg's sign; knee jerks are slightly exaggerated." 


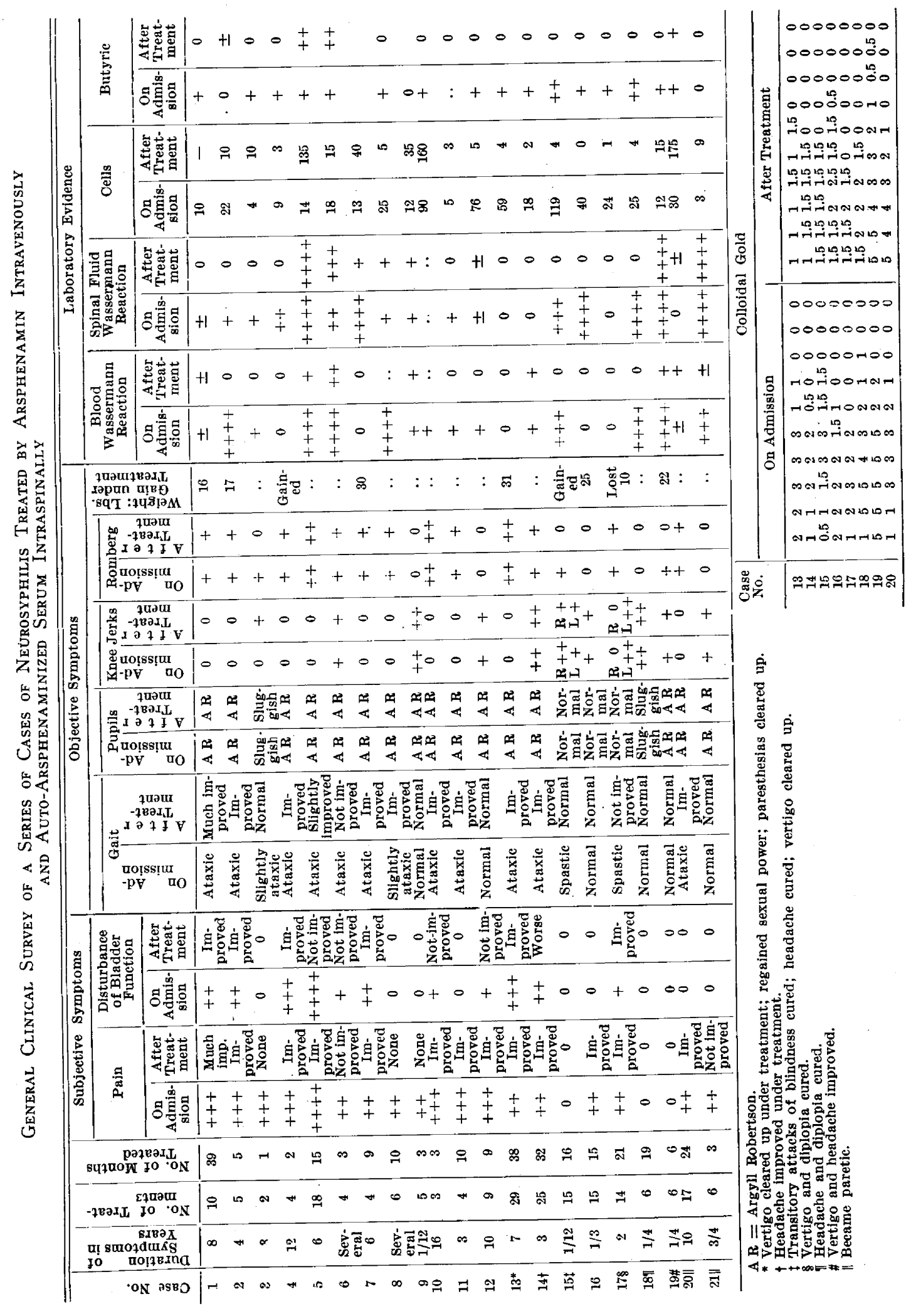

Downloaded From: http://archneurpsyc.jamanetwork.com/ by a University of Calgary User on 05/27/2015 
The foregoing case is interesting because of the extremely severe reaction to one of the treatments and the improvement which occurred later, notwithstanding this reaction.

CASE 4.-History.-M. S., a woman, aged 40. Duration of symptoms twelve years. She has had attacks about twice a month of sharp shooting pains in lower part of back, radiating down sides of abdomen and into legs. Pains associated with nausea and vomiting. Recently gait has been so unsteady that she has been confined to her bed. She has frequent incontinence.

Physical Examination.-This showed a thin, pale, emaciated woman, legs wasted and thin, muscles relaxed. The right pupil was smaller than the left, neither pupil reacted. Knee jerks absent; area of diminished sensation in patch on anterior surface of right thigh, on lower anterior surface of left thigh, and on anterior surface of left leg.

Laboratory Findings on Admission.-Blood Wassermann reaction, negative; spinal fluid Wassermann, ++ ; cell count, 9 ; globulin test, positive.

Treatment.-She received four combined treatments in six weeks. At the end of this time her blood Wassermann reaction remained negative; the spinal fluid showed a negative Wassermann reaction, negative globulin test, and a cell count of 3 .

Results.-This patient improved steadily after the institution of treatment. Interviewed in May, 1916, eleven months after her discharge from the hospital, she had gained much weight, and had partially resumed her housework. Pains still occurred but at much longer intervals, were of less severity, and unassociated with nausea or vomiting. Her bladder control was normal, and her gait, while still ataxic, was much improved, as evidenced by the resumption of her household duties. In reply to a letter of recent date, three years after the last interview, she stated that the improvement noted then had been sustained.

CASE 13.-History.-J. G., a man, aged 45, had symptoms that dated back three years. He complained of severe shooting pains in back and legs, unsteady gait, poor bladder control, and loss of sexual power.

Physical Examination.-Pupils equal, but react sluggishly ; gait ataxic ; marked Romberg sign; has to walk with two canes.

Laboratory Findings.-The blood and spinal fluid Wassermann reactions were negative; globulin test in spinal fluid, negative; cell count, 7 .

Treatment.-This patient has been under treatment for thirty-eight months, and has received twenty-nine combined intravenous and intraspinal treatments. On his last admission in March of this year, the patient reported that he had had very little pain in the last three years; his sexual power had been normal for the past nine months; his bladder gave him very little trouble, and he had gained 31 pounds since beginning treatment. On admission his gait had been so ataxic that he had difficulty in walking with two canes without assistance. At present he uses only one cane, and can walk for a short distance without it.

The foregoing case has shown negative serologic tests throughout. Some observers are of the opinion that intraspinal therapy should be resorted to only in the presence of positive findings in the spinal fluid. We doubt very much if the same results could have been accomplished in this case by intravenous therapy alone. 
CASE 15.-History.-W. B., a man, aged 29, complained of attack of unconsciousness one month before admission with subsequent transitory attacks of blindness; frequent attacks of vertigo and almost constant headache; weakness of the right leg. He had had a chancre and rash five years previously.

Physical Examination. - This revealed normal pupils with a right lateral nystagmus; convergence normal. All deep reflexes exaggerated on the right; ankle clonus and positive Babinski obtained on the right; Romberg positive; spastic gait with dragging of right leg.

Laboratory Findings.-The blood Wassermann reaction was +++ ; spinal fluid Wassermann, +++ ; cells, 119; globulin test, ++ ; colloidal gold curve, 05115231515000 .

Treatment.-This case received twelve injections of mercury at the time of the secondary eruption. On admission the patient was put on mixed treatment and was given two intravenous injections of arsphenamin, $0.6 \mathrm{gm}$. each, and two intraspinal injections of concentrated arsphenaminized serum at intervals of two weeks. He was then treated as an outpatient, being admitted to the hospital once a month for intraspinal injections-fifteen in all. During the intervals he was given mixed treatment.

Results.-At the end of fifteen months this patient is now pursuing his trade as an electrician. The subjective symptoms have entirely disappeared; gait and reflexes are normal, and the serologic findings are negative. Monthly blood and spinal fluid Wassermann tests have been negative for the last five months. He has gained weight under treatment.

CASE 17.-History.-E. S., a woman, aged 39, complained of gradually increasing weakness of the right arm and leg, diplopia, generalized muscular pains and vertigo; difficulty in starting micturition. Duration two years. Has been married twice; no definite history of infection, although the patient believes her first husband had syphilis. One child living and well-later, one miscarriage.

Physical Examination.-This revealed normal pupils, right external strabismus; right knee jerk absent, left exaggerated; Romberg sign positive; gait spastic, bilateral Babinski reflex; no disturbance of sensations.

Laboratory Findings. - The blood and spinal fluid Wassermann reactions were negative; cell count, 24 ; globulin test, positive; colloidal gold curve, 123222000000 .

Treatment.-The patient remained in the hospital two months. During that time she received mixed treatment and two combined intravenous and intraspinal injections. She is now being treated as an outpatient, having received a total of fourteen treatments in twenty-one months.

Results.-At the latest visit she reported marked improvement with a returning use of right arm and leg. She does light housework. Gait still spastic; there is marked improvement in pain, vertigo, and bladder control. Has lost 10 pounds in weight. No strabismus; knee jerks and Romberg sign unchanged. Spinal fluid cells and globulin reaction are now normal, and colloidal gold curve is improved.

CASE 20.-History.-C. V., a man, aged 37, had symptoms during a period of ten years. He has had frequent attacks of pains in legs, and severe attacks of vomiting, unassociated with pain. Legs weak and gait unsteady. Vision in the left eye was poor. 
Physical Examination.-This revealed Argyll Robertson pupils, absence of knee jerks, a moderate Romberg sign, well marked ataxia of lower extremities, and slight ataxia of hands.

Laboratory Findings on Admission.-The blood Wassermann reaction was doubtful, probably positive; spinal fluid Wassermann, negative; cell eount, 30; globulin reaction, positive.

Treatment.-This patient received seventeen combined treatments in two years. Under the treatment his pains became less frequent and less severe in character and there was apparent improvement in his gait. A month before his last admission he began to develop delusions of grandeur, and on admission he was unmistakably paretic and had to be transferred to another institution.

The foregoing case is interesting in that treatment was begun at least a year and a half before the patient became of paretic type and that paresis developed in spite of the treatment.

\section{CONCLUSICNS}

In this series of cases 214 intraspinal treatments have been given. Severe reactions have occurred, particularly in Case 3 , but we have seen no permanent injury or ill effects result from the intraspinal use of auto-arsphenaminized serum.

The treatment has uniformly been of benefit in its effect of increasing the patients' comfort by lessening the severity and frequence of their pains, in frequently improving bladder control, and in improving their nutrition. Improvement in station and in gait has frequently been observed in these cases, and in some, to a very marked extent. 